\title{
Simulation of Electrical Discharge Machine (EDM) Process Pulse Generator Profile
}

\author{
Achebe, P.N. \\ Department of Electrical and Electronic Engineering, Chukwuemeka Odumegwu Ojukwu University, Uli, \\ Nigeria
}

Abstract - Micro Electrical Discharge Machine (EDM) process efficiency is relatively related to the pulse generator, which is the process input. The paper focused on studying the performance of the pulse generator profile of EDM process by carrying out computer simulation in MATLAB/SIMULINK environment. A circuit is obtained for a pulse generator and mathematical equations obtained. Simulations are performed for gap current and gap voltage of EDM pulse generator profile.

Keywords - Computer simulation, EDM, Gap current, Gap voltage, Pulse generator

\section{INTRODUCTION}

Electrical Discharge Machining (EDM) is a controlled metal removal process that involves machining by repeated melting and removal of work piece parts with the use of electric spark erosion [1]. Electric discharge machining provides an effective manufacturing technique that enables the production of parts made of special materials with complicated geometry which are difficult to produce by conventional machining processes [2]. The EDM process requires no mechanical contact between the electrodes and work piece.

The working principle of EDM process is based on the thermoelectric energy created between a workpiece and an electrode submerged in a dielectric fluid with the passage of electric current [2]. The thermoelectric energy is created by the electrical discharge between the workpiece and the electrode as a result of the ionization and momentary breakdown of the dielectric fluid. This is basically caused by the electrode-to-workpiece spark gap voltage. A given small gap separates the workpiece and electrode. This is called spark gap. The voltage/potential difference across the spark gap (between the tool electrode and the workpiece) is known as the spark gap voltage. During the whole process, erosion is produced by electrical discharge due to spark breakdown, voltage falls and abrupt current rises that generate numerous random charges/sparks.

The EDM process starts off with open-gap voltage and as the electrode gets close to the workpiece, it creates an intense electromagnetic field that attracts and aligns ions in the dielectric, the workpiece and the electrode. As the gap continues to get smaller, more ions are attracted and polarized. This reduces the resistivity of the dielectric until the potential voltage can overcome the dielectric's resistivity and current can cross the gap through an ionized path or discharge column. Current is generated and machining begins. As the current increases and voltage decreases, Spark erosion begins.

Withdrawing the potential difference makes the plasma unsustainable. As the plasma channel collapses, it generates pressure or shock waves, which evacuates the molten material forming a crater of removed material around the site of the spark [3]. Hence, the electrons strike the workpiece leading to crater formation due to high temperature and melting and material removal. In the same way, the tool wears as the positive ions impact on it. In EDM, the generator is used to apply voltage pulses between the tool and the workpiece. A constant voltage is not applied. With arcing leading to removal of localised material at a certain point, sparks on the other hand spreads all over the surface of the tool which results to evenly distribution of material removal under the tool [3].

Some parameters that affects the EDM process include discharge voltage, frequency of current, gap between tool electrode and workpiece, ignition delay time, pulse on time, pulse off time, properties of dielectric fluid, conductivity of electrodes, eroding area etc. These process parameters can be classified as Electrical parameters and Non-electrical. The Electrical parameters are Pulse Duration (pulse on time) which is the period current is allowed to pass through the electrode towards the work material within a short gap known as spark gap; Pulse Interval (pulse off time) which is the period of zero current through the electrode; electrode gap (spark gap) which is the distance between the electrode and the workpiece during the EDM process, conductivity of electrodes, eroding area and dielectric fluid. The non-electrical parameters are melting point of the workpiece, the rate of evacuation of the molten material (crater) form between the electrode and the workpiece etc. 
High precision and quality machining is very vital for excellent performance in most modern industries. The EDM ensures that this is achieved as it has the following properties: EDM can process any material that has electric conductivity, it can easily machine materials such as quenched steel and carbide mainly used for tools and difficult to machine by using cutting tools. Also EDM is a non-contact machining technique. It maintains a gap between the electrode and the workpiece in order to ensure electro-discharge between them; therefore, machining can be done without applying pressure on the material [4]. This makes the EDM machining technique very suitable for Micro pit machining (micromachining).

The objective of this paper is to study the EDM pulse generator performance. As such the rest of the paper will focus on the modelling, simulation and conclusion.

\section{ELECTRICAL DISCHARGE MACHINING PULSE GENERATOR EQUATION}

The efficiency of micro EDM process model is known to be closely related to the process input. The input of a typical micro EDM process is a pulse generator. Fig. 1 is the schematic circuit of EDM pulse generator.

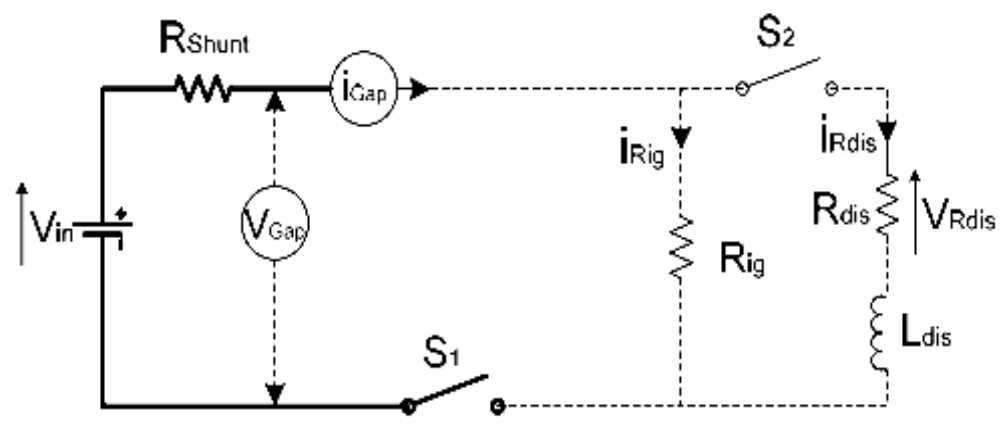

Fig. 1: Circuit of EDM Pulse Generator [5].

The following parameters are defined from the circuit of EDM pulse generator as presented in Table 1.

\begin{tabular}{|c|c|c|}
\hline $\mathbf{S} / \mathbf{N}$ & Definition & Symbol \\
\hline 1 & Shunt resistor & $R_{\text {shunt }}, R_{s}$ \\
\hline 2 & Ignition resistance of the dielectric & $R_{i g}$ \\
\hline 3 & Discharge resistance of the dielectric & $R_{\text {dis }}$ \\
\hline 4 & Ignition current & $i_{\text {Rig }}$ \\
\hline 5 & Discharge gap current & $i_{G a p}$ \\
\hline 6 & Discharge current & $i_{R d i s}$ \\
\hline 7 & Input voltage & $V_{\text {in }}$ \\
\hline 8 & Discharge gap voltage & $V_{G a p}$ \\
\hline 9 & Discharge voltage & $V_{\text {Rdis }}$ \\
\hline 10 & Discharge gap induction & $L_{d i s}$ \\
\hline 11 & Pulse determining switches & $S_{1}$ and $S_{2}$ \\
\hline
\end{tabular}

The mathematical equations describing the dynamics of the pulse generator circuit are obtained by applying Kirchhoff's current and voltage laws (KCL and KVL) with the switch $S_{1}$ closed and the switch $S_{2}$ open.

The gap current dynamic is given by:

$i_{\text {Gap }}=i_{\text {Rig }}+i_{\text {Rdis }}$

The gap voltage is derived given by: 


$$
V_{\text {Gap }}=\frac{R_{i g}}{R_{\text {shunt }}+R_{i g}} V_{\text {in }}
$$

During the discharge phase, $S_{1}$ is closed and $S_{2}$ is closed, the gap voltage can be expressed as follows:

$$
V_{\text {Gap }}-i_{\text {Rdis }} R_{d i s}=L_{\text {dis }} \frac{d i_{\text {Rdis }}}{d t}
$$

Equation (3) can be further simplified given by:

$$
\frac{d t}{L_{d i s}}=\frac{d i_{R d i s}}{V_{G a p}-i_{R d i s} R_{d i s}}
$$

Taking the integration of Eq. (4) gives:

$$
\frac{t}{L_{d i s}}=\int_{0}^{i} \frac{d i_{R d i s}}{V_{G a p}-i_{R d i s} R_{d i s}}
$$

The gap current in the discharge phase can be expressed in the form given by:

$$
i_{\text {Gap }}=\frac{V_{\text {Gap }}}{R_{\text {ig }}}+\frac{V_{\text {Gap }}}{R_{\text {dis }}}\left(1-e^{-\frac{R_{\text {dis }}}{L_{d i s}}}\right)
$$

The MATLAB Simulink model of the EDM pulses in terms of ignition and discharge timing is presented in Fig. 2 . The input voltage is $100 \mathrm{~V}$. The chosen input voltage is sufficient enough to provide significant current for small EDM application. A Mosfet transistor is used in EDM system as a switch to make the output pulses.

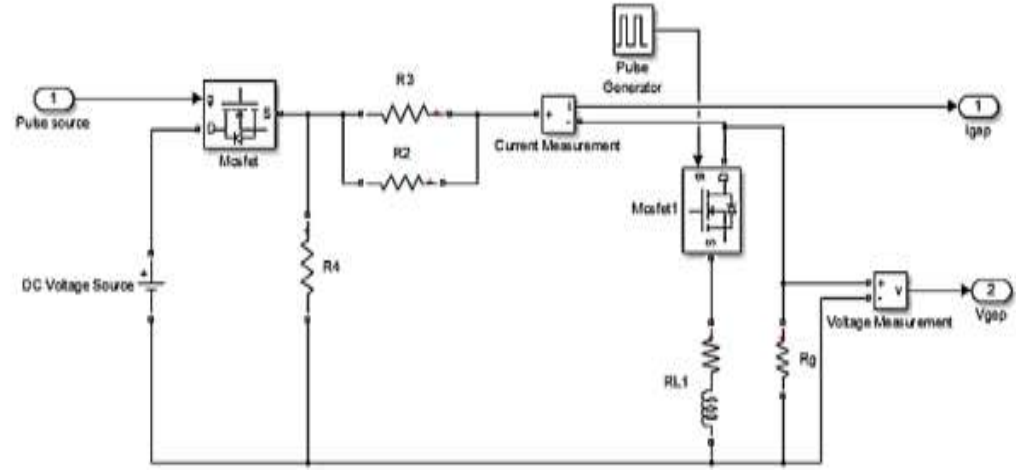

Fig. 2 EDM pulse generator Simulink circuit

\section{A. Material Removal Rate model}

The mathematical Material Removal Rate (MRR) model is developed using dimensional analysis to examine the most effective parameters on the material removal rate or efficiency of the machining [6]. Equation 3.42 represents the MRR.

$$
V^{\&}=C \times \alpha \times V_{G a p} \times i_{G a p} \times F_{s} \times t_{\text {on }}
$$

According to [6], $C$ is an experimental dimensionless constant, $\alpha$ is a material properties factor, $V_{G a p}$ represent gap voltage, $i_{\text {Gap }}$ is gap current, $t_{\text {on }}$ represent discharge pulse on-time, and $F_{s}$ is represent sparking frequency. The parameters are provided in Table 2.

Table 1 Tuned Parameters

\begin{tabular}{|l|l|l|l|}
\hline S/N & Definition & Symbol & Value \\
\hline 1 & Gap Voltage & $V_{\text {Gap }}$ & $25 \mathrm{~V}$ \\
\hline
\end{tabular}




\begin{tabular}{|l|l|l|l|}
\hline 2 & Maximum voltage & $V_{\max }$ & $160 \mathrm{~V}$ \\
\hline 3 & Pulse ON-time & $t_{\text {on }}$ & $0.00000110 \mathrm{~s}$ \\
\hline 4 & Pulse OFF-time & $t_{\text {off }}$ & $0.0000042 \mathrm{~s}$ \\
\hline 5 & Gap current & $i_{\text {Gap }}$ & $25 \mathrm{~A}$ \\
\hline 6 & Sparking Frequency & $F_{S}$ & $8.605 \mathrm{Khz}$ \\
\hline 7 & Material properties factor & $\alpha$ & $2.0 \mathrm{e}-12$ \\
\hline 8 & Dimensional constant & $C$ & 1.74 \\
\hline 9 & Breakdown model parameters & $n$ & 6.57 \\
\hline 10 & Time constant filter & $t$ & 0.00155 \\
\hline
\end{tabular}

\section{B. Breakdown model}

The breakdown model is specifically used to calculate ignition delay time. The ignition delay time is simple the time that elapses before the discharge voltage is attained. Equation 3.44 can be used to represent the ignition delay time [7].

$t_{D}=v \times \delta^{n}$

where $v$ is a flush velocity constant, $\delta$ is the gap width which is the error between the electrode position and the workpiece surface position, and depends on the output of the servomechanism $n$ is the power of the gap width. The conventional parameter values of the breakdown model include: $v=1.04 \times 10^{25}$ and $n=6.57$. Equation (8) becomes:

$t_{D}=1.04 \times 10^{25} \times \delta^{6.57}$

\section{Voltage Average Gap Model}

The average gap voltage is used for the regulation of the gap between electrode and workpiece. It is applied as a measure of the EDM spark gap. An average gap voltage is calculated according to the following equation [7]:

$$
V_{G a p_{-} a v}=\frac{\left(V_{\max } \times t_{D}\right)+\left(V_{G a p} \times t_{o n}\right)}{t_{o n} \times t_{o f f} \times t_{D}}
$$

\section{A. Simulation Results}

\section{RESULTS AND DISCUSSION}

The EDM spark gap Pulse Profile based on pulse power generation are presented. Each of the figure (Fig. 3 and Fig. 4) shows the effect of the spark gap current or voltage on the performance of the EDM process.

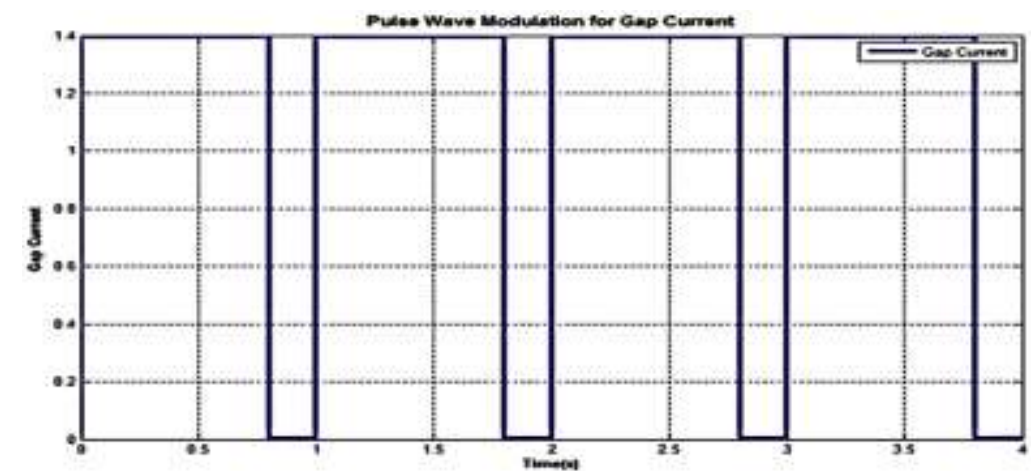

Fig. 3 Pulse profile of EDM process gap current phase 


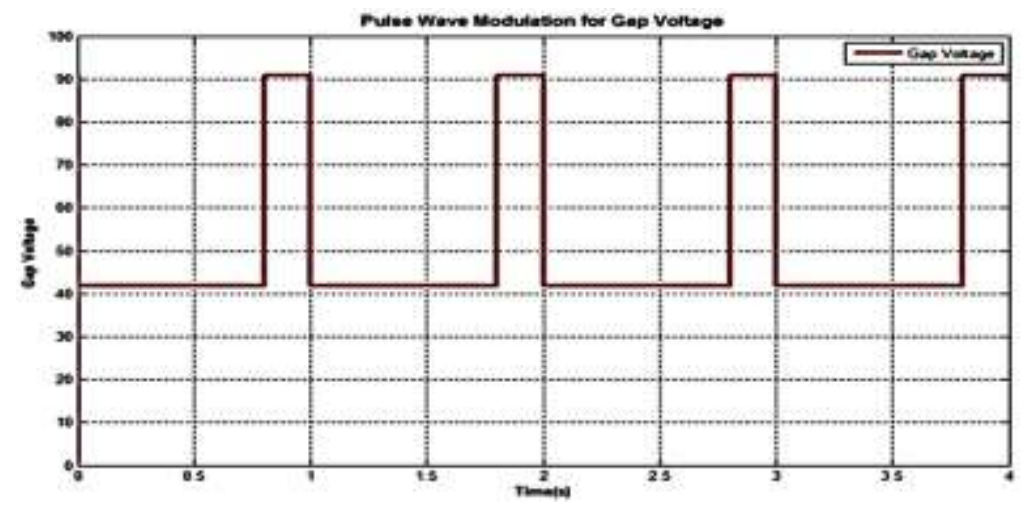

Fig. 4 Pulse profile of EDM process gap voltage phase

\section{B. Discussion}

The EDM process pulse profile during the open circuit state the output gap voltage and gap current are zero. This means that the position of the electrode and the workpiece are in non-discharge position, proceeding the ignition phase. In the ignition delay phase ( 0.5 to 0.82 second). In the pulse on time, a strong electric field is established between electrode and workpiece the period from 0.5 to 0.82 second. This determines the ignition delay time. The ignition delay time is kept constant to ensure the stability of the EDM process. If ignition delay time is too long, this means an open circuit and a prolonged machining process.

Finally in the discharge phase ( 0.82 to 1.0 second) the electrode moves very close to the workpiece and initializes the ionization of the dielectric fluid. Eventually, the electric discharge occurs and the discharge gap voltage drops after which the entire machining process starts all over again.

\section{CONCLUSION}

The paper has presented performance simulation of Electrical Discharge Machine (EDM) process pulse generator profile. A circuit design of the pulse power generator was simulated using MATLAB Simulink software and the profile of voltage and current during machining process was identified based on current and voltage gap. The mathematical model of EDM pulses has been validated by identifying the Power generator Pulse Profile of the EDM Process.

\section{REFERENCES}

[1] (Size 8) S. M. Metev and V. P. Veiko T, Andromeda, ans S. Samion PID Controller Tuning by Differential Evolution Algorithm on EDM Sevo ontrol System, 287, 2266-2270, 2013. http://doi.org/10.4028/www.scientific.net/AMM.284-287.2266

[2] B. Chandra, and H. Singh, "Machining of aluminium metal matrix composites with Electrical discharge machining - A Review." Materials Today: Proceedings, 2(4-5), 1665-1671, 2015. http://doi.org/10.1016/j.matpr.2015.07.094

[3] I. I. T. Kharagpur, "Machining Electro Discharge Machining.” Version 2 ME, IIT Kharagpur, 2012.

[4] C. Chung, S. Chao, and M. F. Lu, "Modeling and Control of Die-sinking EDM," 8(6), pp 713-722, 2009.

[5] E. M. Ade, H. H. K. Nor, Azli Yahya, A. K. N. Trias, F. L. Amorim, amd W. L. Weingaertner, "Die-Sinking Electrical Discharge Machining of a High-Strength Copper- Based Alloy for Injection Molds," XXVI(2), pp 137-144, 2004

[6] L. Hassan, Y. Azli, K. N. Hisham, S. Shahrullail, "Nonlinear Servomotor in Single Pulse Simulation of Electrical Discharge Machining System Modeling,"Faculty of Electrical Engineering, Universiti Teknologi Malaysia, Skudai 81310, Johor, Malaysia, 1(2), pp 319 - 328. 2016.

[7] A. Yahya, and C. D. Manning, "Modelling, Simulation and Controller Design for Electro Discharge Machine System," Faculty of Electrical Engineering, Universiti Teknologi Malaysia, 81310, Skudai, Johor, Malaysia (2), 21-23, 2003 